\title{
Low-energy positron collisions with water: elastic and rotationally inelastic scattering
}

\author{
K.L. Baluja ${ }^{\dagger}$, Rui Zhang*, Jan Franz* and Jonathan Tennyson* \\ ${ }^{\dagger}$ Department of Physics and Astrophysics, University of Delhi, Delhi 110007, India \\ *Department of Physics and Astronomy, University College London, Gower Street, \\ London WC1E 6BT, UK
}

\begin{abstract}
.
Differential, integral and momentum transfer cross sections for the vibrationally elastic and rotationally inelastic scattering of positrons from water at low collision energy $(\mathrm{E} \leq 10 \mathrm{eV})$ are reported. Several models within the $R$-matrix method are used to compute the body-fixed $T$-matrices while the scattering calculations are performed within the fixed-nuclei approximation corrected with the standard Bornclosure formula. These calculations are compared with experimental results for elastic scattering and the best model give reasonable agreement with the most recent measurements (Zecca et al, J. Phys. B, 39, 1597 (2006)) but not with some earlier studies. The relative contribution of the rotationally inelastic processes is investigated and comparisons are made with the equivalent electron scattering cross sections.
\end{abstract}

PACS numbers: $34.85 .+\mathrm{x}$

\section{Introduction}

Water is an extremely important molecule: it is ubiquitous in the universe and fundamental to life. Collisions of positrons with water molecules thus play an important role in a variety of research fields including bio- and medical physics. Water has a large permanent dipole moment and detailed analysis of electron collision measurements (Faure et al. 2004b) suggest that, except in very special cases (Curik et al. 2006), experiments have difficulty determining total elastic and rotationally inelastic cross sections for low-energy collisions because of the very strong forward scattering peak.

Positron-molecule collision measurements are inherently much more difficult than those involving electrons. It is therefore to be anticipated that measurements of lowenergy elastic (and rotationally inelastic) positron-water cross sections should suffer from problems similar to those encountered in the electron measurements. There have been a number of recent attempts to measure elastic cross sections for positronwater collisions which give significantly different results (Sueoka et al. 1986, Sueoka

et al. 1987, Kimura et al. 2000, Zecca et al. 2006, Beale et al. 2006). There are only a limited number of positron - water scattering calculations (Baluja \& Jain 1992, Gianturco et al. 2001, Nishimura \& Gianturco 2004). The most comparable 
previous theoretical study of low-energy positron-water collisions (Gianturco et al. 2001) was primarily concerned with annihilation and therefore did not make corrections for the long-range nature of the positron-dipole interaction. It would therefore appear timely to consider the elastic scattering problem theoretically.

R-matrix calculations of low-energy electron collisions with water (Faure et al. 2004a, Faure et al. 2004b) give excellent agreement with measured differential cross sections for this system, momentum transfer cross sections and, indeed, the recently measured low-energy total cross sections (Curik et al. 2006). It is probably legitimate to claim that the associated calculated elastic and rotationally inelastic cross sections are more reliable than the measured ones in the 1 to $7 \mathrm{eV}$ range.

Despite the absence of exchange, positron molecule collisions are more difficult to treat than the analogous electron molecule collisions. This is because the attractive nature of the interaction between the scattered positron and the electrons in the target leads to polarisation effects in the collision which are hard to model without explicit inclusion of the positron - electron coordinate in the wave function (see Armour \& Humberston (1991) for example). However, the strongly dipolar nature of the water molecule suggests that this system may be easier to study than the non-polar species that have largely been the focus of earlier positron-molecule calculations using the Rmatrix method (Tennyson 1986, Danby \& Tennyson 1990, Danby \& Tennyson 1991).

In this paper, we report calculated differential, integral and momentum transfer cross sections for vibrationally elastic positron scattering from water at collision energies up to $10 \mathrm{eV}$. The water molecule is described by an $a b$ initio multicentred wave function and the scattering calculations are performed using $R$-matrix method within the fixednuclei (FN) approximation. A comparison with the most recent experimental results is presented. The theoretical treatment is briefly introduced in the next section, and details of the models used are given in section 3. Results are discussed in section 4. Our conclusions are summarised in section 5 .

\section{Theory}

The R-matrix method is based on the division of space into an inner region, here defined as a sphere of radius $10 \mathrm{a}_{0}$ centred on the water centre-of-mass, and an outer region. In the inner region, positron-electron correlation effects can be very important. In this region the total wave function can be written:

$$
\Psi_{k}=\sum_{i j} a_{i j k} \phi_{i}\left(x_{1}, \ldots, x_{N}\right) u_{i j}\left(x_{p}\right)+\sum_{i} b_{i k} \chi_{i}\left(x_{1}, \ldots, x_{p}\right),
$$

where $x_{p}$ is the coordinate representing the positron; $\phi_{i}$ are $N$-electron target wave functions; $u_{i j}\left(x_{p}\right)$ are continuum orbitals and $\chi_{i}$ are quadratically integrable $\left(L^{2}\right)$ functions constructed from the target occupied and virtual molecular orbitals. These last functions are important both for relaxing the orthogonality between the target and continuum orbitals, and for representing short-range polarisation effects not included in the truncated close-coupling expansion. It should be noted that in the calculations 
reported below the positron and the electrons share a common orbital set although, of course, the positron will be allowed to occupy orbitals already fully occupied by electrons. The use of a common orbital set for the electrons and the positron has certain computational advantages but is not actually a requirement of our code.

Previous studies of positron-molecule collisions using the R-matrix code have only considered diatomic targets (Tennyson 1986, Danby \& Tennyson 1988, Danby \& Tennyson 1990, Danby \& Tennyson 1991) which were represented using Slater Type Orbitals (STOs). To study collisions on polyatomic molecules we have re-programmed the UK polyatomic R-matrix package (Morgan et al. 1997, Morgan et al. 1998), which is based on the use of Gaussian Type Orbitals (GTOs) to represent both target and continuum wave functions, to allow for positron collisions. As before (Tennyson 1986), this involved separately identifying the kinetic and Coulomb one-electron integrals so they could be treated with the appropriate signs and ensuring that exchange integrals involving positrons were all set to zero. A complication which needed to be addressed is that the codes have a new, highly efficient, algorithm for Hamiltonian matrix construction (Tennyson 1996a) which has not previously been used for positron scattering. The new code was extensively tested using positron collisions from diatomics, particularly $\mathrm{H}_{2}$, and from acetylene. Results from our preliminary study of acetylene will be reported elsewhere (Franz et al. 2007).

In the outer region, the positron moves in the scattering potential given by the target multipole moments. These are similar to the corresponding electron moments once adjusted for the changed charge of the positron, except that positrons do not couple target states of different spin symmetries. This means that for water only singlet target states need to be included in the close-coupling expansion, eq. (1).

For strongly dipolar molecules such as water it is necessary to compensate for the truncated partial wave expansion used in the expansion of the positron wave function. This situation is well known from electron scattering problems (Lane 1980, Gianturco \& Jain 1986, Morrison 1988) where the standard procedure is to use the dipole Born approximation to correct for this truncation.

In the present work, two distinct approaches were tested for this. In the first a simple Born correction using an approximate closure formula was applied to the partial cross sections and hence to the total elastic cross sections following the approach of Baluja et al. (2000). In the second approach, the T-matrices were initially subjected to a frame-transformation to the laboratory frame and the Born correction applied to the resulting rotationally-resolved T-matrices. The latter cross sections, which are mostly those reported below, were computed using the program POLYDCS of Sanna \& Gianturco (1998). The quantitative differences between the two methods are shown in figure 1.

Above $1 \mathrm{eV}$ the two methods gave similar results although, of course, the frametransformation approach gives additional information in terms of rotationally resolved cross sections. This approach should also be the more reliable one at the lower energies and is therefore used below, where we only consider transitions from the $J=0$ ground state of water. This is because Okamoto et al. (1993) showed that the differential cross 
section (DCS), $\frac{d \sigma}{d \Omega}$, does not depend on the initial rotational state of the target molecule unless the scattering angle is very close to $0^{\circ}$. This means that theoretical DCS can be computed for the target in its ground rotational state, independent of the (rotational) temperature of the corresponding experiment. It would only appear to be necessary to average over a particular rotational state distribution at energies close to rotational thresholds, where the adiabatic nuclei rotation (ANR) approximation becomes invalid (Morrison 1988). At present positron beams do not allow sufficient energy resolution to study such near threshold processes.

\section{Calculations}

All calculations were performed at the equilibrium geometry of $\mathrm{H}_{2} \mathrm{O}, r_{\mathrm{OH}}=1.81 a_{0}$ and $\widehat{H O H}=104.5^{\circ}$ (Csaszar et al. 2005). This means that all cross sections presented correspond to the vibrationally elastic ones.

Given the known sensitivity of positron scattering to polarisation effects and the difficulty, in general, of reproducing these using close-coupling expansions of the type employed here, we performed the calculations using several independent models to give a test of the robustness of our result. For all models the continuum orbitals were represented using the GTO basis of Faure et al. (2002) which includes partial waves up to $l=4$ and is optimised to span energies below $20 \mathrm{eV}$.

To test our models we used two distinct target GTO basis sets for $\mathrm{H}$ and $\mathrm{O}$ atoms. The smaller of these was a DZP set which gives 15 molecular orbitals of $a_{1}$ symmetry, 5 of $b_{1}, 10$ of $b_{2}$ and 3 of $a_{2}$, which we denote $(15,5,10,3)$. Of these $(3,1,1,0)$ are occupied in the ground state of water and the rest our virtual. The second basis used the DZP GTOs of Dunning Jr (1970a) for O and TZ basis of Dunning Jr (1970b) for H with an added diffuse s function and two p functions (Gorfinkiel et al. 2002); this gives an orbital space of $(19,6,12,3)$. Within the self-consistent field (SCF) approximation the smaller basis gives a permanent target dipole moment of $1.986 \mathrm{D}$ whereas the larger one gives $1.983 \mathrm{D}$. This latter value is improved to $1.897 \mathrm{D}$ within the NO model described below, close to the experimental value of 1.854 D (Suresh \& Naik 2000). All results below were computed with the larger basis.

In our simplest model the target was frozen in its ground configuration and the positron was allowed to occupy all target and continuum orbitals of the appropriate symmetry. This model is designated static below.

Polarisation effects were included in the static plus polarisation (SP) model augmenting the static model with a large number of excited states. A 125 singlet excited target states of $\mathrm{A}_{1}, \mathrm{~B}_{1}, \mathrm{~B}_{2}$ and $\mathrm{A}_{2}$ symmetries, 49, 24, 39, 13 respectively, were generated by allowing all possible single excitations of a target electron into the full virtual state specified above. Again the positron was then allowed to occupy all target and continuum orbitals of the appropriate symmetry. For electron scattering such models must be used with caution because of the danger of introducing target (extra) correlation effects into the scattering calculation (Tennyson 1996b). However 
the distinct nature of the scattering positron removes this risk.

Our third model, labelled NOs below, introduced polarisation effects in a fashion more usual in electron scattering calculations (Gorfinkiel et al. 2002, Gorfinkiel et al. 2005). In this model we used four target states in the close-coupling expansion (1), a singlet of each symmetry. State-averaged Natural Orbitals (NOs) were generated by performing a singles and doubles configuration interaction (SD CI) calculation starting from the complete active space (CAS) which was given by $\left(2 \mathrm{a}_{1}, 3 \mathrm{a}_{1}, 4 \mathrm{a}_{1}, 5 \mathrm{a}_{1}, 1 \mathrm{~b}_{1}, 2 \mathrm{~b}_{1}\right.$, $\left.1 b_{2}\right)^{8}$. State averaging was performed for the lowest singlet state of each symmetry with the ground $\mathrm{X}^{1} \mathrm{~A}_{1}$ state weighted eight times more than the other states. The scattering calculation used these four target states in the close coupling expansion (1) and each state was represented by a CAS SD CI model. A CAS singles CI model for the target states was also tested but gave similar results. In the scattering calculation the positron was allowed to occupy all continuum orbitals and the $(6,2,1,0)$ target orbitals.

\section{Results and discussion}

Figure 2 compares rotationally summed elastic cross sections for positron-water as a function of the different models discussed above. It can be seen that the dominant nature of the water dipole means that the results are actually rather insensitive to the degree of polarisation included in the model. Indeed the static, SP and NO models all give rather similar results. Conversely including the Born correction leads to a very significant increase in our calculated cross sections, particularly at low collision energies.

Figure 2 also compares our calculations with the previous study of Gianturco et al. (2001). Our Born-corrected results give very good agreement with the results reported in this earlier work. Superficially this is surprising since Gianturco et al did not employ a Born correction in their calculation. However, their single centre method leads to many more partial waves being used in their wave function expansion and it would appear that they included enough to obtain converged results.

Since our various models, once Born corrected, give very similar results all further results reported are for the NO model although in each case these results were checked using our other models. Figure 3 presents differential cross sections (DCS) positronwater scattering at 2 and $5 \mathrm{eV}$. As is expected for a dipolar system, these DCS are strongly forward peaked. Looking at the rotationally resolved partial cross sections, it is apparent that the DCS are also completely dominated by the $\Delta J=1$ component. This is true at all energies between 1 and $10 \mathrm{eV}$ and it is only for the DCS at $0.25 \mathrm{eV}$ that the magnitude $\Delta J=0$ component become at all comparable with the $\Delta J=1$ one, and then only for sideways scattering which is disfavoured at all energies. This behaviour differs significantly from the electron-water scattering results of Faure et al. (2004b) which find $\Delta J=0$ to be much more important and even find that neglecting $\Delta J=2$ also leads to poor results.

Figure 4 compares our total, rotationally summed cross section (Born corrected NOs model) with the various published measurements of this process. There is a wide scatter 
in the experimental results which is hardly surprising given the importance of forward scattering in this problem and the difficulty of performing reliable measurements at low angles (Cho et al. 2003). Indeed none of three measurements lying below our curve either correct for the effect of missing low angle scattering events or, indeed, give an acceptance profile for their positron scattering measurements. Knowledge of the latter would allow detailed theoretical modelling of observations via the DCS, as has been done for the electron case (Curik et al. 2006, Jones \& Field 2007). Given the constraints on the experimental measurements one would expect uncorrected experiments to underestimate the elastic cross sections, particularly at the lower scattering energies. It can be seen from figure 4 that these three measurements do indeed lie systematically below our calculations. We consider these measurements (Sueoka et al. 1987, Zecca et al. 2006, Beale et al. 2006) to be consistent with our predictions,

Kimura et al. (2000) did correct the earlier measurements of Sueoka et al. (1987) for the effects of forward scattering using a procedure applied to other dipole dominated scattering problems (Okamoto et al. 1993, Hamada \& Sueoka 1994). This procedure is based on use of the unmodified dipole-Born approximation which, for positron-water, significantly overestimates the forward scattering contribution to the cross section, particularly at low energies. It is not surprising therefore that their cross sections are significantly higher than, particularly below $2 \mathrm{eV}$. We attempted to repeat this analysis using our cross sections but unfortunately the procedure applied is not well enough specified for us to repeat it.

Figure 5 gives our rotationally resolved cross sections as a function of energy. As with the DCS these are dominated by the $\Delta J=1$ transition, although the rotational elastic $\Delta J=0$ does increase in importance at low energies. We note that our results should be treated with caution at energies approaching the rotational excitation thresholds since the adiabatic nuclei approximation we use treats all thresholds as degenerate which cannot be assumed to be a suitable approximation at very low energies, although recent studies show that it work surprisingly well (Faure et al. 2006, Jones $\&$ Field 2007). Figure 5 also makes comparison with the equivalent total electron cross sections of Faure et al. (2004b). The electron cross sections are somewhat higher although they appear to converge to the same value at very low energies. Since pure dipole scattering for positrons and electrons would give the same cross sections, these differences are a reflection of how polarisation effects modify the dipole potential.

Finally figure 6 presents our momentum transfer cross sections which are compared with the equivalent cross sections for electron collisions of Faure et al. (2004b). Faure et al's momentum transfer cross sections agree well with measured ones (Cho et al. 2003) as these measurements are not sensitive to the problematic with forward angles. We note that the electron momentum transfer cross sections are higher than the positron ones at all energies considered. 


\section{Conclusions}

We report rotationally resolved elastic cross sections for low-energy positron scattering from water using various models. Once Born corrected to allow for effects introduced by the large permanent dipole moment of water, our results show surprising little dependence on model and hence on the level of treatment of the polarisation. This is in strong contrast to positron scattering from non-dipolar molecules and must be a consequence of the dominant effect of the dipole potential.

There have been a number of measurements of elastic scattering of positrons by water but such studies are hampered by the fact that such collisions are strong forward scattered and a general lack of knowledge about the acceptance angle of the various experiments. Our results lie between the two measurements by Sueoka and co-workers (Sueoka et al. 1987, Kimura et al. 2000) and somewhat above those of Zecca et al. (2006). Given that none of the experimental results have been corrected for neglected forward scattering we would expect our results to be somewhat higher than the measurements. This suggests that the results of Kimura et al. (2000) are somewhat too high. Our results are consistent with the other measurements (Sueoka et al. 1987, Zecca et al. 2006, Beale et al. 2006). Until progress is made with the experimental acceptance angle problem, we would suggest that our calculations give the most reliable values for the positron-water elastic cross section at energies below the positronium formation threshold.

\section{Acknowledgments}

We thank Nella Laricchia and Edward Armour for helpful discussions and support from EPSRC and the Royal Society under their India-UK exchange programme. 


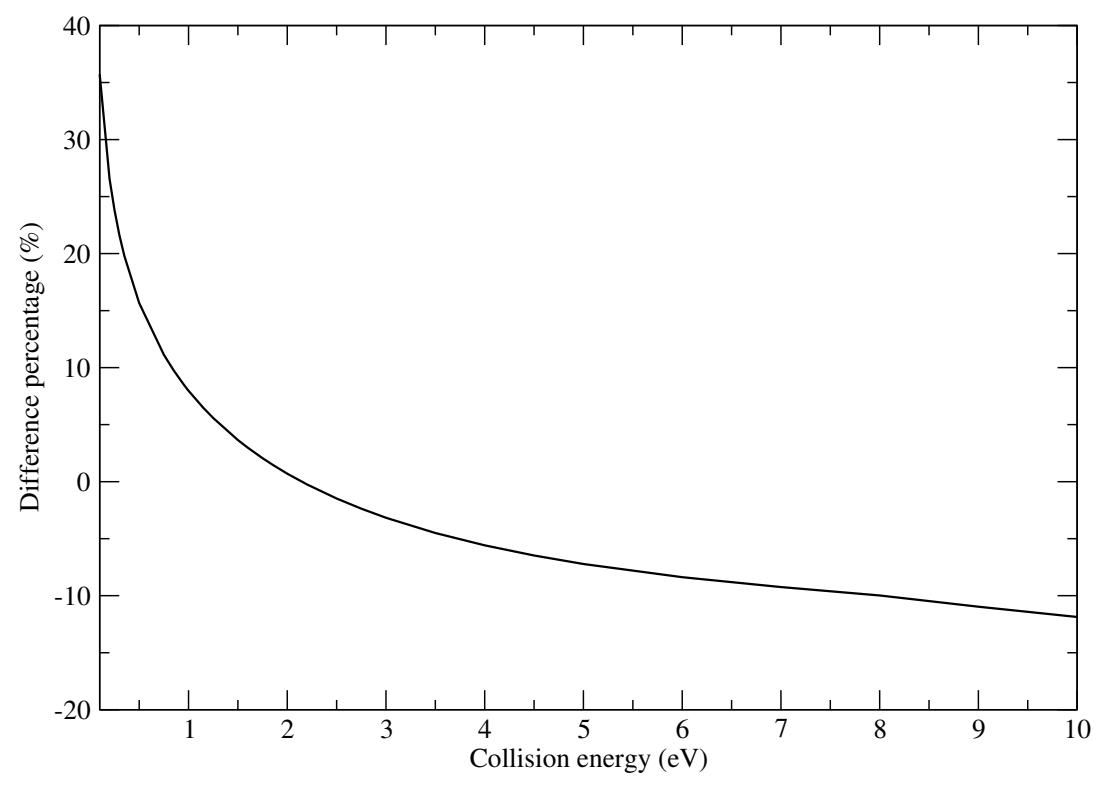

Figure 1. Comparison of the dipole Born corrections given by the frame transformation correction of the DCS calculated POLYDCS (Sanna \& Gianturco 1998) and direct Born correction of the elastic cross section. The difference is defined as percentage difference between POLYDCS and the simple Born calculation. Results are for the NO model, although very similar comparisons are obtained for other models.

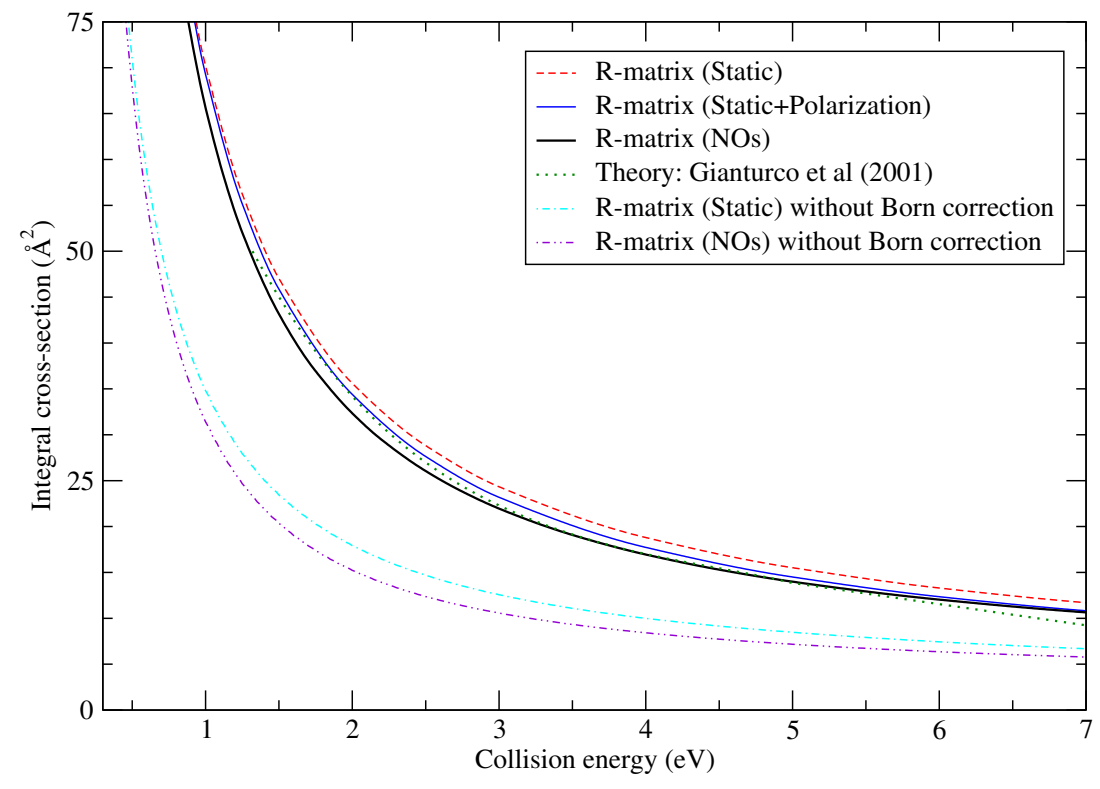

Figure 2. Calculated elastic (rotationally summed) integral cross sections for positronwater collisions as a function of the collision energy for several theoretical models, see text for details.

\section{References}

Armour E A G \& Humberston H W 1991 Phys. Rep. 204, 165-251. 

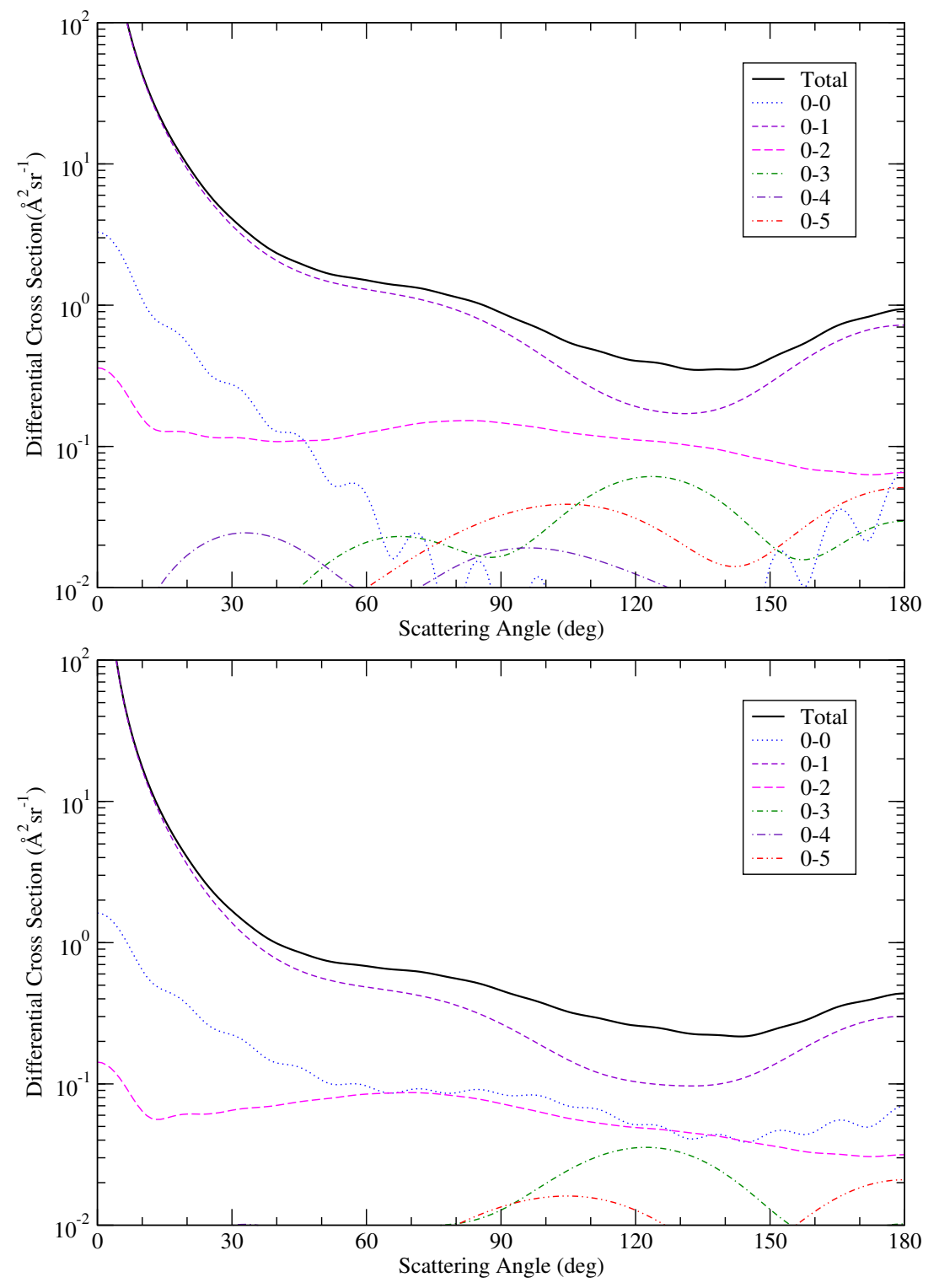

Figure 3. Differential cross section (DCS) for positron-water collisions at $2 \mathrm{eV}$ (upper) and $5 \mathrm{eV}$ (lower). The solid line gives the total DCS while the other lines give the rotationally resolved partial state-to-state DCS.

Baluja K L \& Jain A 1992 Phys. Rev. A 45, 7838-7845.

Baluja K L, Mason N J, Morgan L A \& Tennyson J 2000 J. Phys. B: At. Mol. Opt. Phys. 33, L677L684.

Beale J, Armitage S \& Laricchia G 2006 J. Phys. B: At. Mol. Opt. Phys. 39, 1337-1344.

Cho H, Lee H S \& Park Y S 2003 Radiat. Phys. Chem. 68, 115.

Csaszar A G, Czako G, Furtenbacher T, Tennyson J, Szalay V, Shirin S V, Zobov N F \& Polyansky O L 2005 J. Chem. Phys. 122, 214205.

Curik R, Ziesel J P, Jones N C, Field T A \& Field D 2006 Phys. Rev. Lett. 97, 123202.

Danby G \& Tennyson J 1988 Phys. Rev. Letts. 61, 2737-2739.

Danby G \& Tennyson J 1990 J. Phys. B: At. Mol. Opt. Phys. 23, 1005-1016. erratum 23, 2471 (1990). 


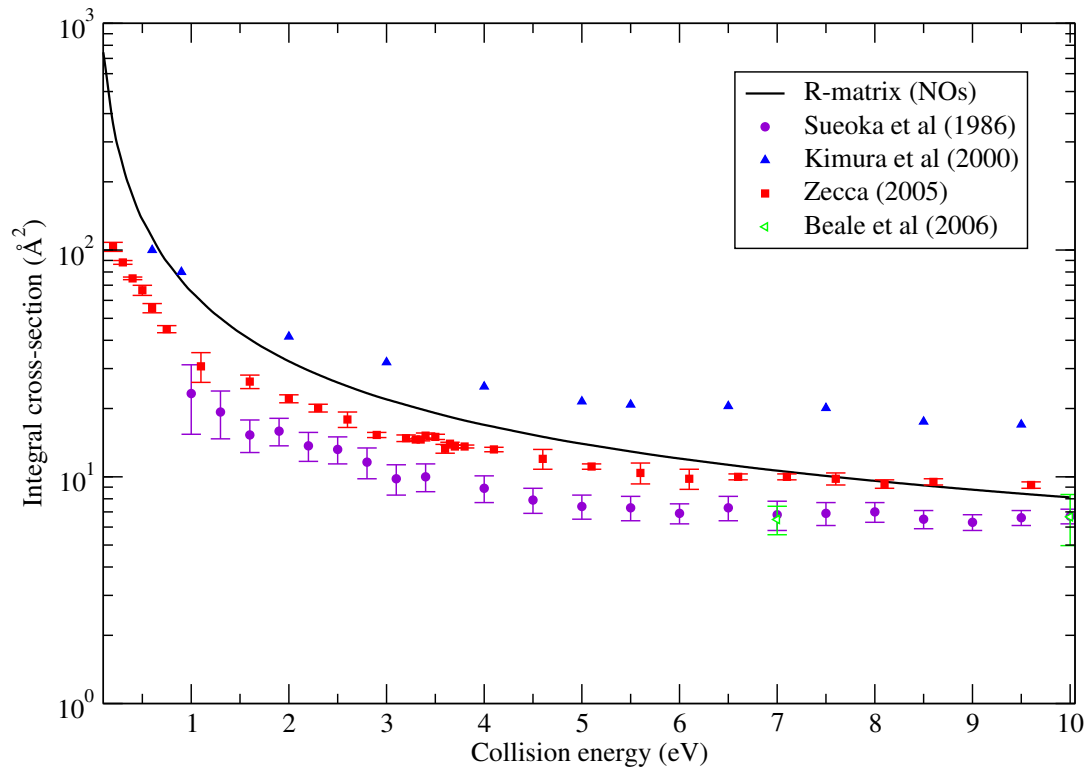

Figure 4. Comparison of experiment with theory for the elastic (rotationally summed) integral cross sections for positron-water collisions as a function of the collision energy.

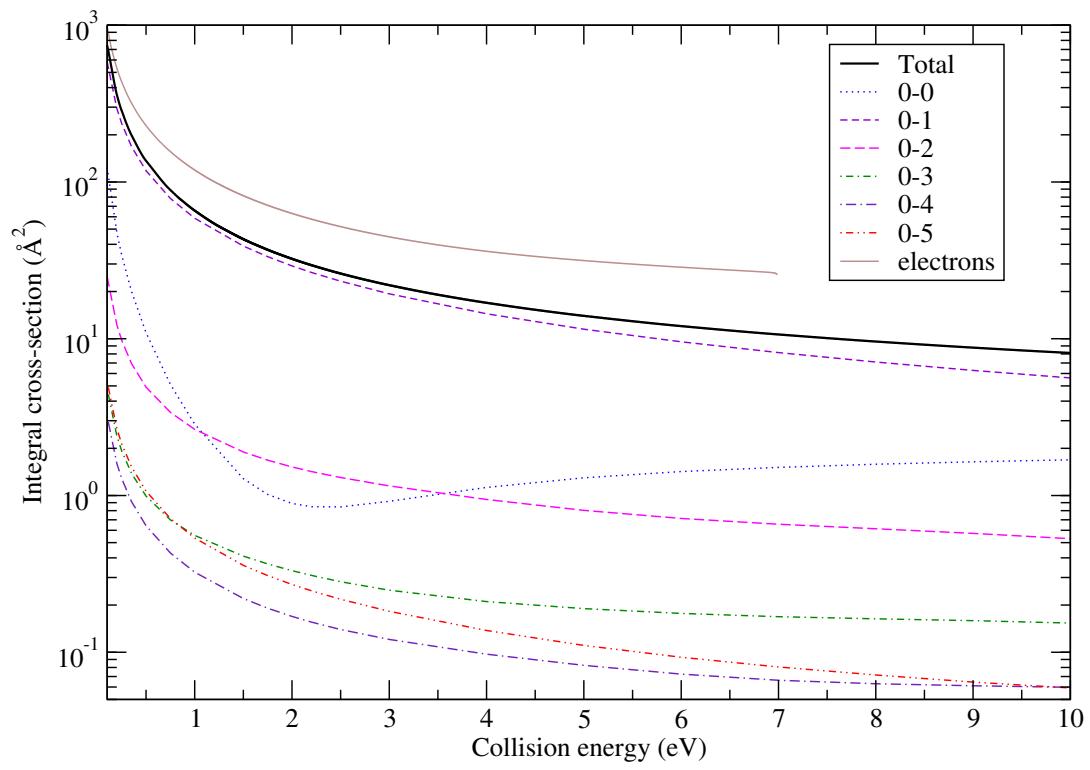

Figure 5. Calculated elastic (rotationally summed) integral cross sections for positronwater collisions as a function of the collision energy compared to the equivalent cross sections for electron collisions (Faure et al. 2004b). Also shown are the rotationally resolved cross sections for positron collisions. 


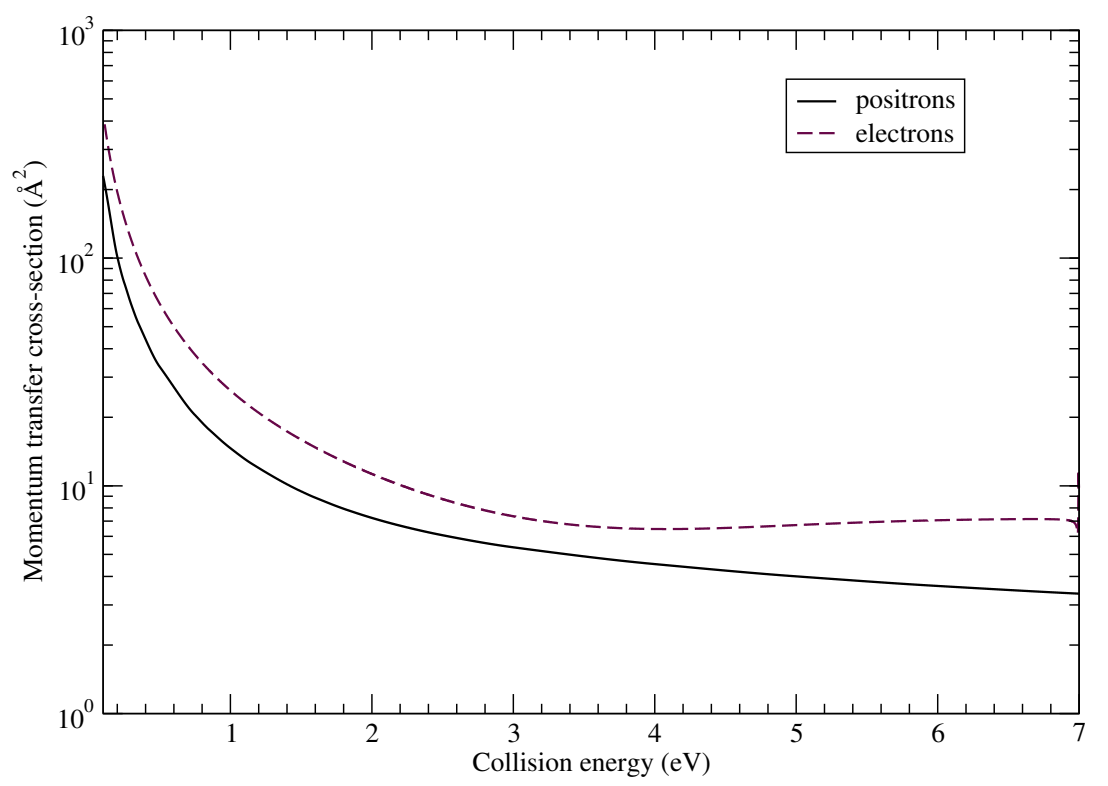

Figure 6. Elastic (rotationally summed) momentum transfer cross sections for position (this work) and electron (from Faure et al. (2004b)) water scattering as a function of the collision energy.

Danby G \& Tennyson J 1991 J. Phys. B: At. Mol. Opt. Phys. 24, 3517-3529.

Dunning Jr T H 1970 a J. Chem. Phys. 53, 2823.

Dunning Jr T H $1971 b$ J. Chem. Phys. 55, 716.

Faure A, Gorfinkiel J D, Morgan L A \& Tennyson J 2002 Computer Phys. Commun. 144, 224-241.

Faure A, Gorfinkiel J D \& Tennyson J 2004a Mon. Not. Roy. astr. Soc. 347, 323-333.

Faure A, Gorfinkiel J D \& Tennyson J 2004b J. Phys. B: At. Mol. Opt. Phys. 37, 801-807.

Faure A, Kokoouline V, Greene C H \& Tennyson J 2006 J. Phys. B: At. Mol. Opt. Phys. 39, 42614273.

Franz J, Baluja K L, Gianturco F A, Tennyson J, Lucchese R R, Gibson T L \& Stoecklin T 2007 in preparation.

Gianturco F A \& Jain A 1986 Phys. Rep. 143, 347-425.

Gianturco F A, Mukherjee T \& Occhigrossi A 2001 Phys. Rev. A 64, 032715.

Gorfinkiel J D, Faure A, Taioli S, Piccarretta C, Halmova G \& Tennyson J 2005 Euro. J. Phys. D 35, 231-237.

Gorfinkiel J D, Morgan L A \& Tennyson J 2002 J. Phys. B: At. Mol. Opt. Phys. 35, 543-555.

Jones N C \& Field D 2007. private communiction.

Hamada A \& Sueoka O 1994 J. Phys. B : At. Mol. Opt. Phys. 27, 5055-5064.

Kimura M, Sueoka O, Hamada A \& Itikawa Y 2000 Advan. Chem. Phys. 111, 537-622.

Lane N F 1980 Rev. Mod. Phys. 52, 29-119.

Morgan L A, Gillan C J, Tennyson J \& Chen X 1997 J. Phys. B: At. Mol. Opt. Phys. 30, 4087-4096.

Morgan L A, Tennyson J \& Gillan C J 1998 Computer Phys. Comms. 114, 120-128.

Morrison M A 1988 Adv. At. Mol. Phys. 24, 51.

Nishimura T \& Gianturco F A 2004 Nucl. Instr. and Meth. in Phys. Res. B 221, 24-29.

Okamoto Y, Onda K \& Itikawa Y 1993 J. Phys. B: At. Mol. Opt. Phys. 26, 745-758.

Sanna N \& Gianturco F A 1998 Comput. Phys. Commun. 114, 142-167.

Sueoka O, Mori S \& Katayama Y 1986 J. Phys. B: At. Mol. Phys. 19, L373-378.

Sueoka O, Mori S \& Katayama Y 1987 J. Phys. B: At. Mol. Phys. 20, 3237-3246.

Suresh S J \& Naik V M 2000 J. Chem. Phys. 113, 9727. 
Tennyson J 1986 J. Phys. B: At. Mol. Phys. 19, 4255-4263.

Tennyson J 1996a J. Phys. B: At. Mol. Opt. Phys. 29, 1817-1828.

Tennyson J 1996b J. Phys. B: At. Mol. Opt. Phys. 29, 6185-6201.

Zecca A, Sanyal D, Chakrabarti M \& Brunger M J 2006 J. Phys. B: At. Mol. Phys. 39, 1597-1604. 